\title{
LAMIACEAE MEDICINALES Y AROMÁTICAS COMERCIALIZADAS EN EL ÁREA METROPOLITANA DE BUENOS AIRES, ARGENTINA
}

\author{
Medicinal and aromatic Lamiaceae commercialized in the Buenos Aires Metropolitan Area, \\ Argentina
}

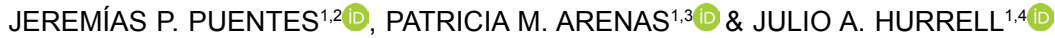

\begin{abstract}
Resumen: Esta contribución, enmarcada en la etnobotánica urbana, presenta 21 especies de Lamiaceae cuyos productos se comercializan como medicinales y aromáticos (condimenticios y saborizantes) en el Área Metropolitana de Buenos Aires, Argentina. Se relevaron 200 sitios de expendio del circuito comercial general (dietéticas) y 5 supermercados del circuito comercial restringido del segmento de inmigrantes chinos. En los sitios de venta se adquirieron muestras de referencia de productos de las distintas especies. Se entrevistaron 410 personas acerca de los usos asignados a cada especie. Para definir estos usos se recurrió también a otras fuentes de información, entre estas, los medios masivos. El trabajo se suplementó con una revisión de los estudios académicos sobre la actividad biológica y efectos evaluados de las especies relevadas, a los fines de su correlación con los usos localmente asignados. La mayoría de las especies relevadas $(87,5 \%)$ son "visibles": se comercializan en el circuito comercial general, lo cual evidencia el nivel elevado de conocimiento botánico local sobre las Lamiaceae. Asimismo, la presencia de las plantas y productos incrementa la diversidad biocultural local, es decir, tanto la diversidad de especies (dimensión biológica) como de sus conocimientos asociados (dimensión cultural).
\end{abstract}

Palabras clave: Conocimiento botánico, contextos pluriculturales, Etnobotánica urbana, productos vegetales, usos locales.

Summary: This study, framed in urban ethnobotany, presents 21 species of Lamiaceae whose products are marketed as medicinal and aromatic (condiments and flavorings) in the Buenos Aires Metropolitan Area, Argentina. Two hundred outlets of the general commercial circuit (health food stores) and five supermarkets of the restricted commercial circuit of the Chinese immigrant segment were surveyed. Samples of products of the different species were acquired in the outlets. Four hundred and ten persons were interviewed about uses assigned to each species. To define these uses, other sources of information were also utilized, including mass media. In addition, a review of the academic studies on the biological activity and evaluated effects of the surveyed species was done in order to link them with the locally assigned uses. The majority of the species surveyed (87.5\%) are "visible": they are sold in the general commercial circuit, which evidences the high level of local botanical knowledge about Lamiaceae. Likewise, the presence of plants and plant products increases the local biocultural diversity, that is, both the diversity of species (biological dimension) and their associated knowledge (cultural dimension).

Key words: Botanical knowledge, local uses, plant products, pluricultural contexts, urban ethnobotany.

\footnotetext{
${ }^{1}$ Laboratorio de Etnobotánica y Botánica Aplicada, Facultad de Ciencias Naturales y Museo, Universidad Nacional de La Plata. Calle 64 nro. 3, 1900-La Plata - CONICET, Argentina.

${ }^{2}$ E-mail: jeremiasppuentes@gmail.com

33E-mail:parenas@fcnym.unlp.edu.ar

${ }^{4}$ E-mail: juliohurrell@gmail.com
} 


\section{Introducción}

La Etnobotánica urbana es una disciplina de desarrollo relativamente reciente, que estudia las relaciones entre las personas y las plantas en los contextos pluriculturales urbanos. En particular, estudia la composición y la dinámica del conocimiento botánico local en contextos culturalmente heterogéneos. El carácter pluricultural de los escenarios urbanos se basa en la presencia de diversos segmentos de inmigrantes, que introducen tanto productos vegetales como sus saberes y creencias asociados. En este marco, el "conocimiento botánico urbano" es un sistema que se compone tanto de conocimientos no tradicionales (porque los conocimientos llamados "tradicionales" corresponden, por definición, a contextos culturalmente homogéneos), como de conocimientos ligados a las tradiciones de origen de los diversos segmentos de inmigrantes (Hurrell, 2014; Hurrell et Pochettino, 2014).

En el contexto urbano, el conocimiento botánico se difunde según la dinámica de circulación de los productos vegetales en los circuitos comerciales. Diversos productos circulan en el circuito comercial general y resultan, por lo tanto, "visibles" para la mayoría de los habitantes locales. Los productos que circulan solo en los circuitos comerciales restringidos de los grupos de inmigrantes resultan, en cambio, "invisibles" para la mayoría de los habitantes locales. La dinámica del conocimiento botánico urbano se evidencia en aquellos productos del circuito restringido de los inmigrantes que ingresan al circuito comercial general, entonces, productos que eran "invisibles" se vuelven "visibles". Este proceso se denomina "visualización" y es potenciado en gran medida por "agentes de visualización", como los medios masivos (en especial Internet) y las tiendas de alimentos saludables que reciben el nombre local de "dietéticas", y que difunden tanto productos vegetales como sus conocimientos asociados (Hurrell, 2014; Hurrell et Pochettino, 2014).

Estos conceptos forman parte del marco teórico-metodológico de una línea de investigación en Etnobotánica urbana del Laboratorio de Etnobotánica y Botánica Aplicada (LEBA), pionera en nuestro país, desarrollada en el Área Metropolitana de Buenos Aires (AMBA). La presente contribución comprende resultados parciales de esta línea de investigación, referidos a las especies de la familia Lamiaceae, muchas de ellas conocidas y valoradas desde la antigüedad por sus usos medicinales y aromáticos (condimenticios, saborizantes). Esta familia botánica es la tercera en cantidad de especies comercializadas en el AMBA hasta el momento (21 especies), luego de Asteraceae (54 especies) y Leguminosae (38 especies) (Puentes, 2017). En este marco, este trabajo sigue la línea de otro previo, referido a la familia Asteraceae (Hurrell et Puentes, 2013).

\section{Materiales y Métodos}

El área de estudio comprende dos aglomerados urbanos contiguos: 1) el Gran Buenos Aires, conformado en torno a la Ciudad Autónoma de Buenos Aires que, junto a los partidos vecinos de la Provincia de Buenos Aires, constituye el mayor aglomerado urbano del país, tanto en extensión como en población; 2) el Gran La Plata, conformado a partir de la ciudad de La Plata, la capital de la provincia de Buenos Aires, que incluye los partidos de Ensenada, Berisso y La Plata (Fig. 1). Ambos aglomerados constituyen el Área Metropolitana de Buenos Aires (AMBA), con un área total de unos $5000 \mathrm{~km}^{2}$ y cerca de 14 millones de habitantes (Hurrell, 2014; Hurrell et Pochettino, 2014; Puentes, 2017).

En los trabajos de campo se realizaron entrevistas semiestructuradas y abiertas, método de uso habitual en etnobotánica, y el enfoque es cualitativo (Albuquerque et al., 2014; Puentes, 2017), a fin de presentar el inventario de especies de Lamiaceae, sus productos y usos locales. Para esta contribución se relevaron 205 sitios de expendio. De estos, 200 corresponden al circuito comercial general, mayormente a las "dietéticas". La selección de estos 


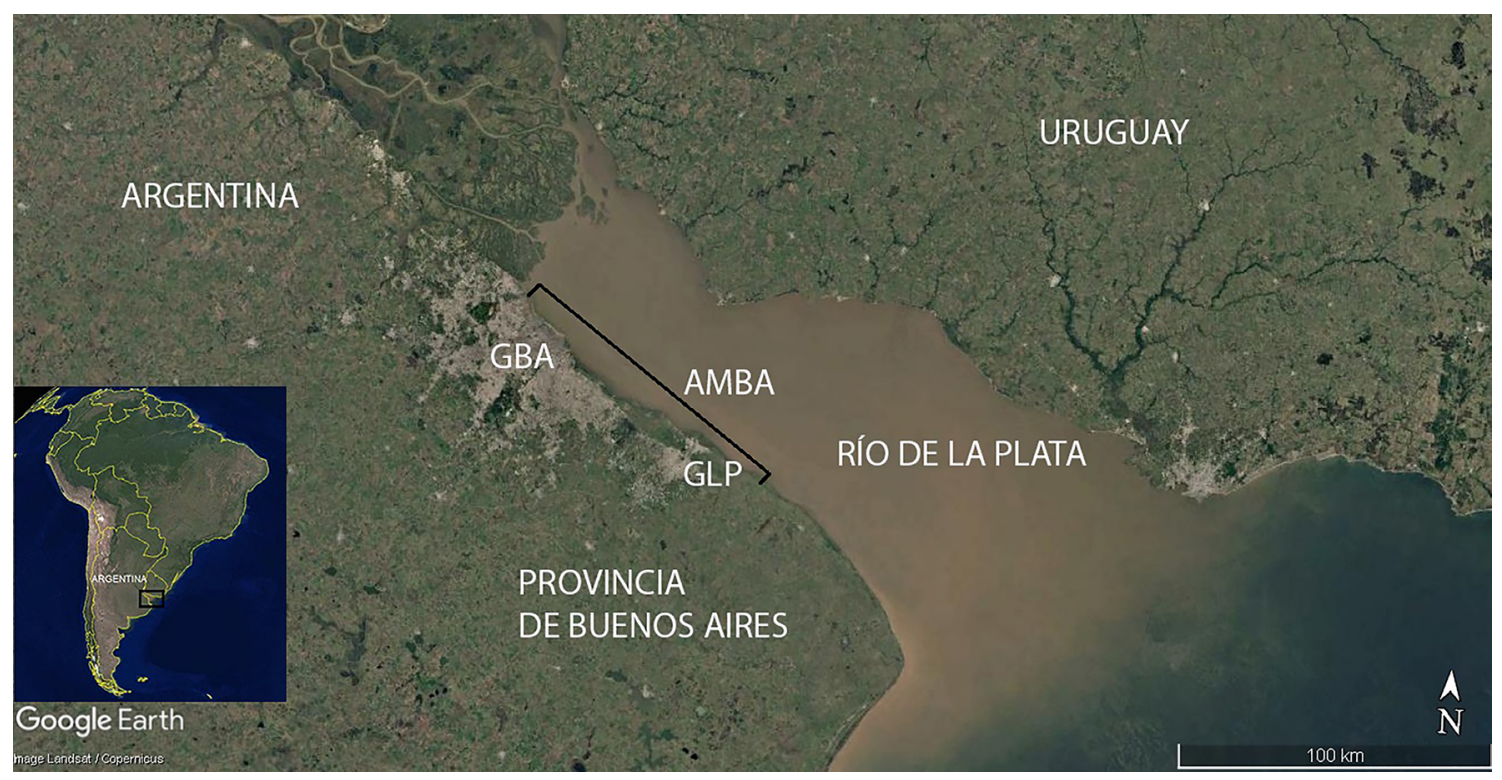

Fig. 1. Área de estudio. AMBA: Área Metropolitana de Buenos Aires; GBA: Gran Buenos Aires; GLP: Gran La Plata (Google Earth).

Fig. 1. Study area. AMBA: Buenos Aires Metropolitan Area; GBA: Greater Buenos Aires; GLP: Greater La Plata (Google Earth).

puntos de venta comenzó al azar y continuó hasta alcanzar la saturación de la información requerida. Los otros 5 sitios de expendio corresponden al total de grandes supermercados del llamado "Barrio Chino", que constituyen el circuito comercial restringido del grupo de inmigrantes chinos en la Ciudad Autónoma de Buenos Aires. Este segmento de inmigrantes ha alcanzado una presencia conspicua en los últimos años y ha introducido productos de especies que aumentan la diversidad biocultural local (tanto de productos como de saberes), en particular, de productos vinculados a la Fitoterapia Tradicional China (Hurrell et Puentes, 2017).

Desde el año 2008 hasta la fecha se realizaron entrevistas, previo consentimiento informado, a 410 personas de ambos sexos y distintas edades, dos por cada punto de venta, seleccionadas entre vendedores $(80 \%)$ y consumidores $(20 \%)$. La duración promedio de cada entrevista fue de aproximadamente 1 hora. En los relevamientos en los sitios de expendio se obtuvieron registros fotográficos y se adquirieron muestras de los productos de las especies evaluadas, que se identificaron con un código alfanumérico y se depositaron en las colecciones etnobotánicas del LEBA para su documentación. Las especies botánicas fueron identificadas mediante sus caracteres morfológicos en el caso de las muestras de materiales frescos y de materiales secos fragmentados (productos de herboristería) o enteros (por ejemplo, semillas). Para otros tipos de muestras (materiales en polvo, tinturas, cápsulas, comprimidos), se consideró la identificación que consta en las etiquetas oficiales de cada producto. Para los nombres científicos actualizados se ha consultado The Plant List (2013).

Los "usos localmente asignados" de las especies relevadas provienen de las entrevistas realizadas, y se complementaron con datos obtenidos en distintas fuentes, como etiquetas y folletos de los productos, y la información que circula en los medios masivos, principalmente Internet, que difunde de forma rápida y en múltiples direcciones a la vez los conocimientos vinculados a las especies, saberes que 
orientan a los consumidores locales en su selección de productos a emplear.

Por último, siguiendo los protocolos del LEBA, el trabajo de campo se suplementó con una exhaustiva revisión bibliográfica sobre la actividad biológica y los efectos estudiados de las especies relevadas en ámbitos académicos, a fin de correlacionarlos con los usos locales asignados. Se revisaron los datos incluidos en artículos disponibles en Google Académico y mayormente en la base de datos PubMed (2019). Este trabajo de revisión es relevante a los fines de este estudio, porque la información relacionada con el conocimiento científico pasa a formar parte de los saberes locales de los consumidores, una vez que son difundidos.

\section{Resultados}

Los resultados obtenidos se resumen en la Tabla 1. En la primera columna se incluyen los nombres científicos actualizados, nombres locales y origen geográfico. Además, se incluyen los productos de los circuitos comerciales y los códigos alfanuméricos de las muestras de referencia (Fig. 2). Las muestras exclusivas del circuito comercial restringido de los inmigrantes chinos se indican con un asterisco. La segunda columna corresponde a los usos localmente asignados, indicándose las categorías generales de uso: medicinales y aromáticos. La tercera columna incluye la actividad biológica y efectos estudiados para las distintas especies; se mencionan tanto trabajos de revisión, que incluyen distintos efectos, como los casos de trabajos referidos a efectos particulares.

Del total de 21 especies relevadas, solo dos son nativas: Clinopodium gilliesii y Minthostachys verticillata; las 19 especies restantes son exóticas, de las cuales seis se encuentran naturalizadas: Marrubium vulgare, Melissa officinalis, Mentha spicata, Mentha $\times$ piperita, Nepeta cataria y Salvia officinalis.

Respecto de las categorías generales de usos asignados, cinco especies se emplean solo con fines terapéuticos: Leonurus japonicus, Nepeta cataria, Orthosiphon stamineus, Prunella vulgaris y Salvia hispanica; las 16 restantes se consideran para ambos fines: terapéuticos y aromáticos (condimentos, saborizantes).

En relación a la visibilidad de las especies relevadas, tres de estas (14,3\%) presentan productos exclusivos de los supermercados del "Barrio Chino": Leonurus japonicus, Perilla frutescens y Prunella vulgaris; por ende, son especies "invisibles" para la mayoría de la población urbana local. Las 18 especies restantes $(85,7 \%)$ son "visibles": se comercializan en el circuito comercial general (dietéticas).

\section{Discusión y Conclusiones}

Como se observa en la Tabla 1, en términos generales hay correlación entre los usos localmente asignados, por un lado, y la actividad biológica y efectos estudiados, por el otro. En ocasiones, esa correlación es directa, por ejemplo, el uso local "adelgazante" se vincula con el efecto estudiado "antiobesidad" (Melissa officinalis, Orthosiphon stamineus, Salvia hispanica); el uso local "antiestrés" se vincula con el efecto "adaptógeno" (Prunella vulgaris, Salvia hispanica); o el uso local "afrodisíaco", que se asocia con los efectos "disfunción eréctil" y "potenciador de la libido" (Clinopodium gilliesii). Otras veces la correlación es indirecta, por ejemplo, en el caso de Hyssopus officinalis, su uso local "antiespasmódico" puede vincularse con efectos como "relajante muscular" y "antiinflamatorio"; y en el caso de Orthosiphon stamineus, su uso "antirreumático" puede asociarse a los efectos "antiinflamatorio" y "analgésico".

La elevada visibilidad de especies relevadas $(85,7 \%)$ indica un elevado conocimiento de las personas sobre las Lamiaceae que circulan en el escenario urbano local, en correlato con la difusión amplia y de larga data de las especies condimenticias, como la albahaca, la lavanda, la melisa, las mentas, el orégano, el romero, la salvia y el tomillo, así mismo valoradas positivamente por sus efectos terapéuticos. Salvia hispanica, la chía, presenta 


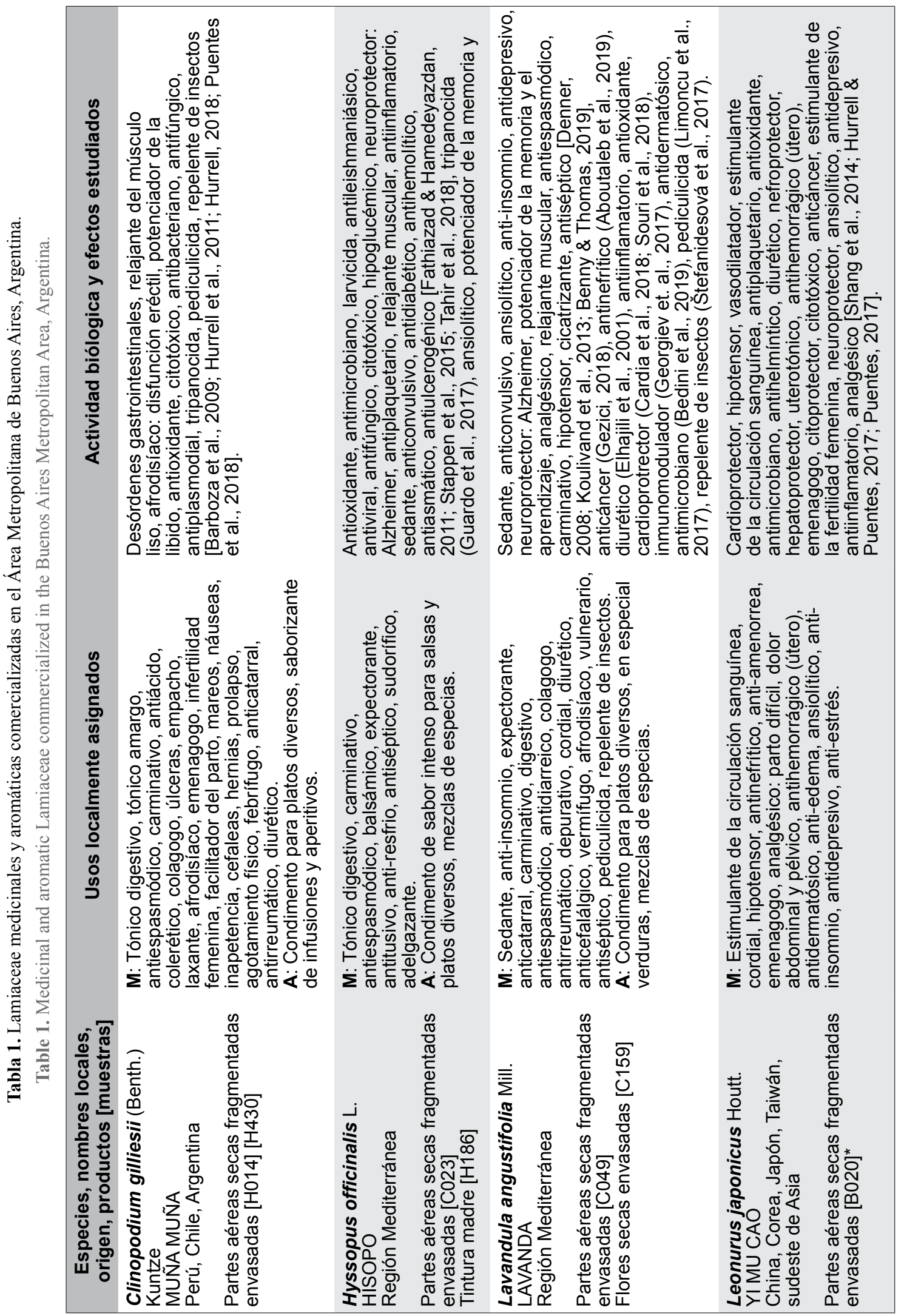




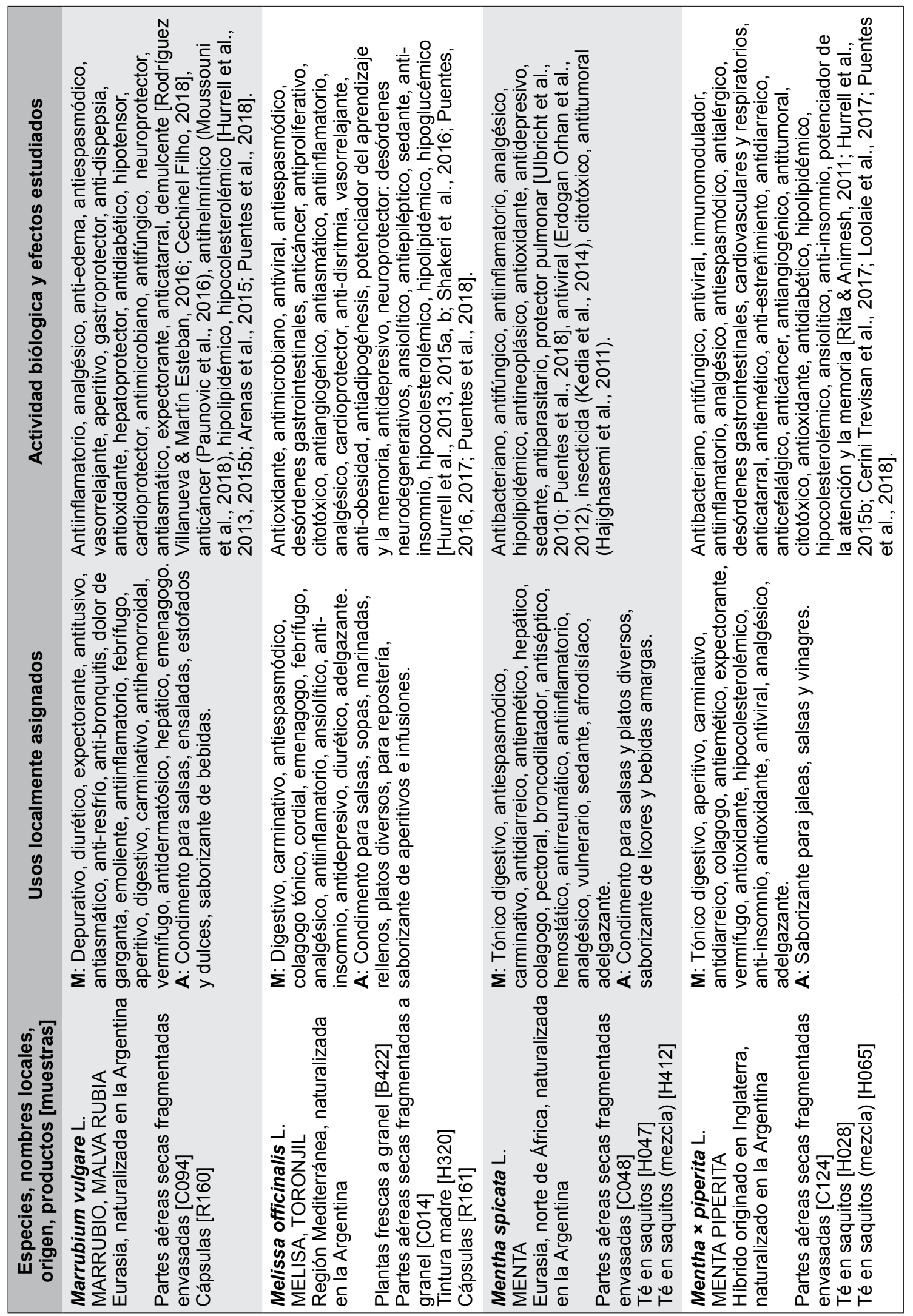




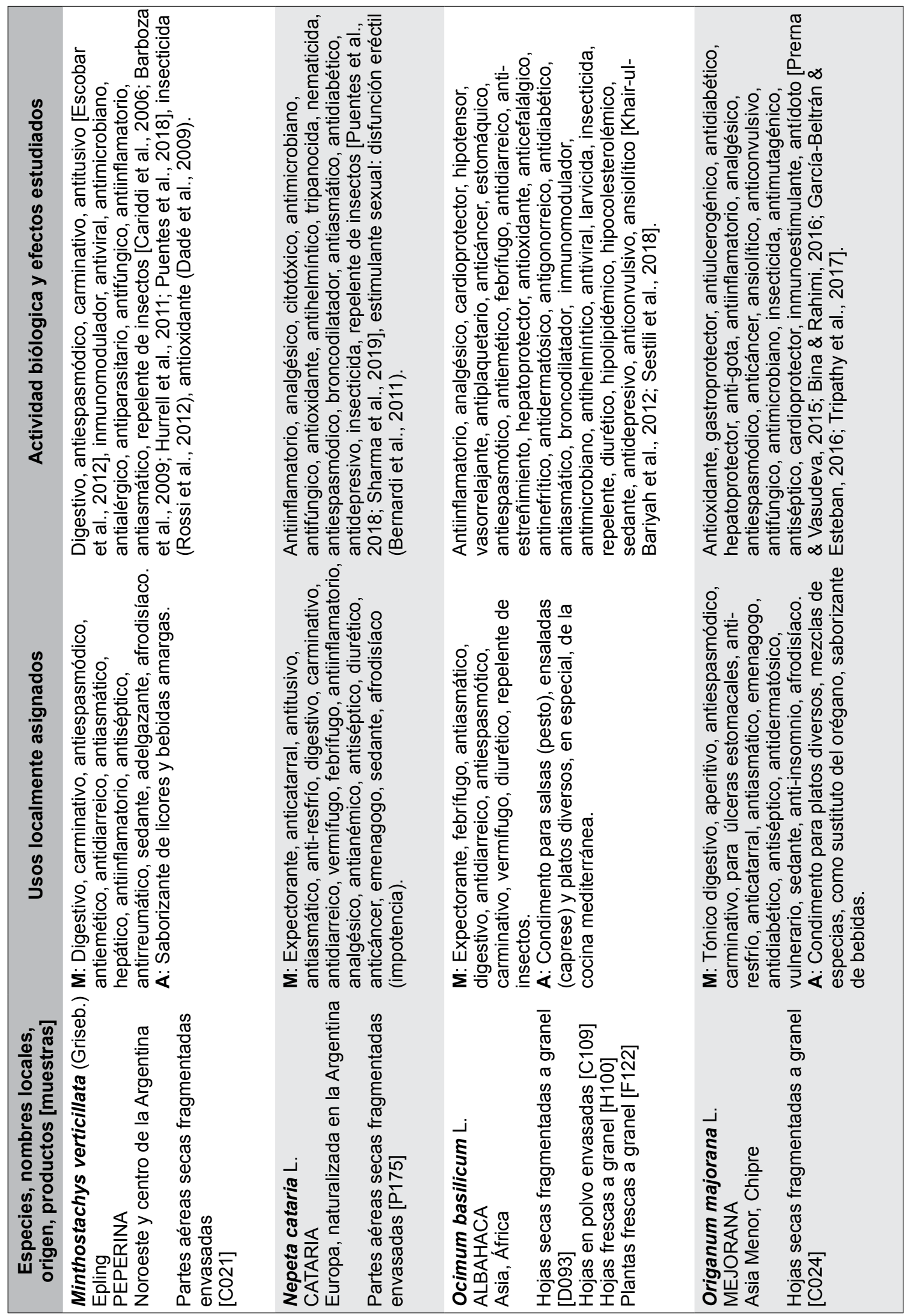




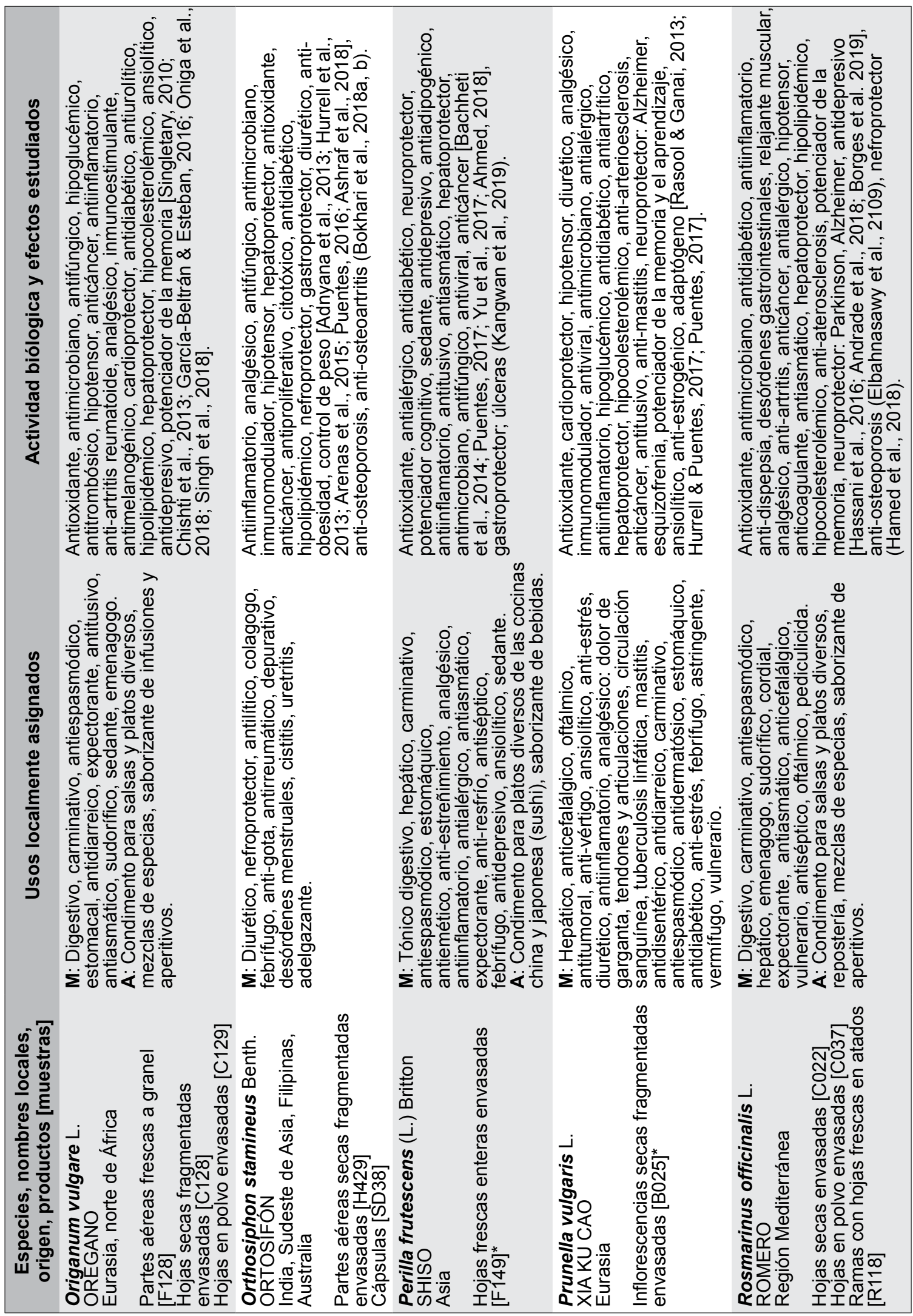




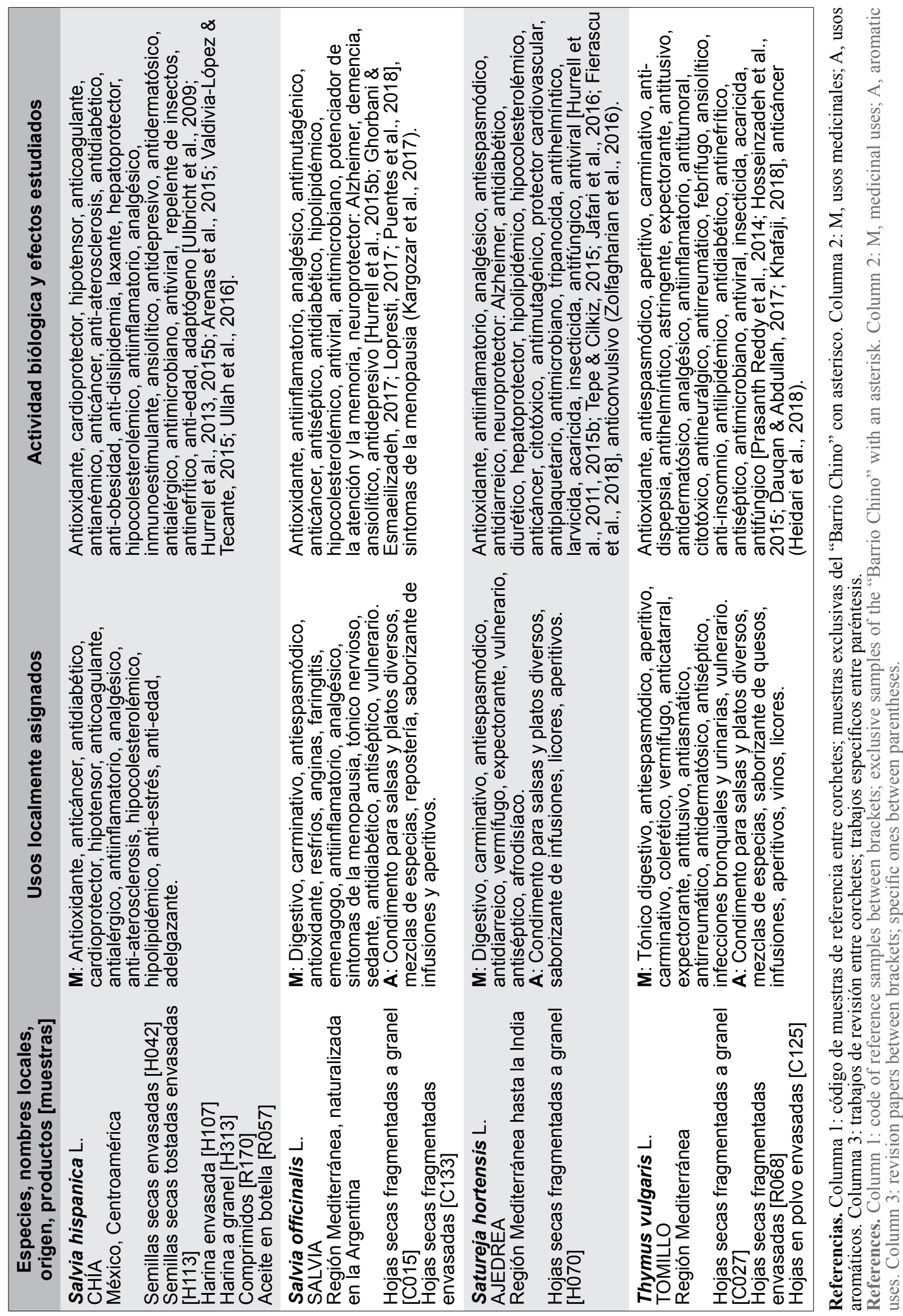




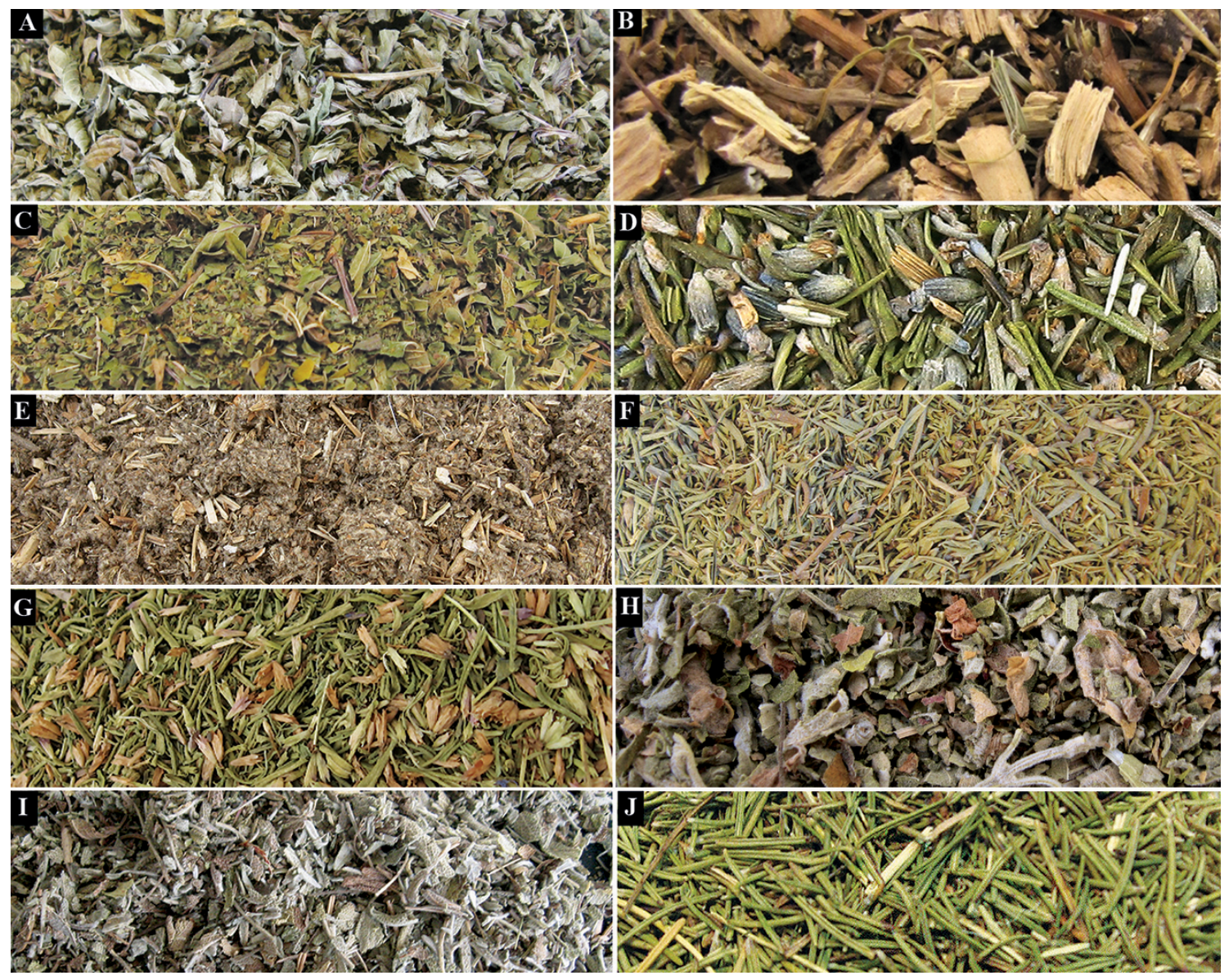

Fig. 2. Muestras de productos de herboristería de algunas de las especies tratadas. A: Minthostachys verticillata (peperina). B: Clinopodium gilliesii (muña muña). C: Mentha spicata (menta). D: Lavandula angustifolia (lavanda). E: Marrubium vulgare (marrubio). F: Satureja hortensis (ajedrea). G: Hyssopus officinalis (hisopo). H: Melissa officinalis (melisa, toronjil). I: Salvia officinalis (salvia). J: Rosmarinus officinalis (romero).

Fig. 2. Samples of herbal products from some treated species. A: Minthostachys verticillata (peperina). B: Clinopodium gilliesii (muña muña). C: Mentha spicata (spearmint). D: Lavandula angustifolia (lavander). E: Marrubium vulgare (white horehound). F: Satureja hortensis (summer savory). G: Hyssopus officinalis (hyssop). H: Melissa officinalis (lemon balm). I: Salvia officinalis (sage). J: Rosmarinus officinalis (rosemary).

una difusión muy amplia pero reciente en los medios masivos, debida principalmente a las aplicaciones terapéuticas de sus semillas, la mayor fuente vegetal de ácidos grasos omega 3 , con efecto hipocolesterolémico, entre otros (Hurrell et al., 2013, 2015b).

En el marco del estudio realizado es importante resaltar que, para el contexto académico, los efectos estudiados validan los usos locales asignados. Sin embargo, para el contexto etnobotánico, los usos locales tienen valor en tanto son identificados y aceptados por los consumidores de productos vegetales, con independencia del ámbito académico. No obstante, muchos habitantes urbanos seleccionan los productos a consumir a partir de los "efectos científicamente validados" (un argumento de venta muy frecuente) que son difundidos por los medios masivos y se incorporan al sistema de conocimiento botánico local.

Esta contribución describe el estado actual de las Lamiaceae comercializadas en el marco del área de estudio, 21 especies con diversos usos asignados, tanto en el ámbito terapéutico 
como en el de la alimentación (aromáticas condimenticias y saborizantes), con un panorama adicional acerca de los estudios realizados hasta el momento sobre la actividad biológica y efectos de cada especie relevada. La presencia de estas especies, a través de distintos productos, incrementa la diversidad biocultural local, es decir, tanto la diversidad de especies como de sus conocimientos asociados. En este marco, esta presentación netamente descriptiva puede constituir una base adecuada para alentar a futuro el desarrollo de estudios de distintos niveles de profundidad.

\section{Agradecimientos}

Los autores agradecen a las personas entrevistadas por su desinteresada colaboración, a la Dra. María Lelia Pochettino, y por su intermedio a los integrantes del LEBA, por su apoyo permanente, y a los revisores anónimos por sus comentarios y sugerencias. La línea de investigación en Etnobotánica urbana del LEBA es desarrollada gracias al soporte financiero de la Universidad Nacional de La Plata y el Consejo Nacional de Investigaciones Científicas y Técnicas (CONICET), Argentina.

\section{Bibliografía}

ABOUTALEB, N., H. JAMALI, M. ABOLHASANI \& H. PAZOKI TOROUDI. (2019). Lavender oil (Lavandula angustifolia) attenuates renal ischemia/reperfusion injury in rats through suppression of inflammation, oxidative stress and apoptosis. Biomedicine and Pharmacotherapy 110: 9-19.

https://doi.org./10.1016/j.biopha.2018.11.045.

ADNYANA, I. K., F. SETIAWAN \& M. INSANU. (2013). From ethnopharmacology to clinical study of Orthosiphon stamineus Benth. International Journal of Pharmacy and Pharmaceutical Sciences 5: 66-73.

AHMED, H. M. (2018). Ethnomedicinal, phytochemical and pharmacological investigations of Perilla frutescens (L.) Britt. Molecules 24 (1): pii: E102. https://doi.org./10.3390/molecules24010102

ALBUQUERQUE, U. P., L. V. F. CRUZ DA CUNHA, R. F. P. LUCENA \& R. R. N. ALVES (eds.). (2014). Methods and techniques in Ethnobiology and Ethnoecology. Springer-Humana Press, New York, USA.
ANDRADE, J. M., C. FAUSTINO, C. GARCIA, D. LADEIRAS, C. P. REIS \& P. RIJO. (2018). Rosmarinus officinalis L.: an update review of its phytochemistry and biological activity Future Science OA 4(4): FSO283.

https://doi.org./10.4155/fsoa-2017-0124

ARENAS, P. M., M. B. DOUMECQ, J. P. PUENTES \& J. A. HURRELL. (2015). Algas y plantas comercializadas como adelgazantes en el Área Metropolitana de Buenos Aires, Argentina. Gaia Scientia 9: 32-40.

ASHRAF, K., S. SULTAN \& A. ADAM. (2018). Orthosiphon stamineus Benth. is an outstanding food medicine: review of phytochemical and pharmacological activities. Journal of Pharmacy \& Bioallied Sciences 10: 109-118.

https://doi.org./10.4103/jpbs.JPBS_253_17

BACHHETI, R. K., A. JOSHI \& T. AHMED. (2014). A phytopharmacological overview on Perilla frutescens. International Journal of Pharmaceutical Sciences Review and Research 26: 55-61.

BARBOZA, G. E., J. J. CANTERO, C. O. NUÑEZ, A. PACCIARONI \& L. ARIZA ESPINAR. (2009). Medicinal plants: A general review and a phytochemical and ethnopharmacological screening of the native Argentine Flora. Kurtziana 34: 7-365.

BEDINI, S., G. FLAMINI, F. COSCI, R. ASCRIZZI, M. C. ECHEVERRIA, E. V. GOMEZ, L. GUIDI, M. LANDI, A. LUCCHI \& B. CONTI. (2019). Toxicity and oviposition deterrence of essential oils of Clinopodium nubigenum and Lavandula angustifolia against the myiasis-inducing blowfly Lucilia sericata. PLoS One 14: e0212576. https://doi.org./10.1371/journal.pone.0212576

BENNY, A. \& J. THOMAS. (2019). Essential oils as treatment strategy for Alzheimer's Disease: Current and future perspectives. Planta Medica 85: 239-248.

https://doi.org./10.1055/a-0758-0188

BERNARDI, M. M., T. B. KIRSTEN, J. H. LAGO, T. M. GIOVANI \& C. O. MASSOCO. (2011). Nepeta cataria L. var. citriodora (Becker) increases penile erection in rats. Journal of Ethnopharmacology 137: 1318-1322. https://doi.org./10.1016/j.jep.2011.07.061

BINA, F. \& R. RAHIMI. (2016). Sweet marjoram: a review of ethnopharmacology, phytochemistry, and biological activities. Journal of Evidence-Based Integrative Medicine 22: 175-185. https://oi.org/10.1177/2156587216650793 
BOKHARI, R. A., S. F. LAU \& S. MOHAMED. (2018a). Orthosiphon stamineus (Misai Kucing) ameliorated postmenopausal osteoporosis in rat model. Menopause 25: 202-210

https://doi.org./10.1097/GME.0000000000000980

BOKHARI, R. A., N. A. TANTOWI, S. F. LAU \& S. MOHAMED. (2018b). Java Tea (Orthosiphon stamineus) protected against osteoarthritis by mitigating inflammation and cartilage degradation: a preclinical study. Inflammopharmacology 26: 939949. https://doi.org./10.1007/s10787-017-0432-2

BORGES, R. S., B. L. S. ORTIZ, A. C. M. PEREIRA, H. KEITA \& J. C. T. CARVALHO. (2019). Rosmarinus officinalis essential oil: a review of its phytochemistry, antiinflammatory activity, and mechanisms of action involved. Journal of Ethnopharmacology 229: 29-45. https://doi.org./10.1016/j.jep.2018.09.038

CARDIA, G. F., S. E. SILVA-FILHO, E. L. SILVA, N. S. UCHIDA, H. A. CAVALCANTE, L. L. CASSAROTTI, V. E. SAlVADEGO, R. A. SPIRONELLO, C. A. BERSANI-AMADO \& R. K. CUMAN. (2018). Effect of lavender (Lavandula angustifolia) essential oil on acute inflammatory response. Evidence-Based Complementary and Alternative Medicine 2018:1413940. https://doi.org./10.1155/2018/1413940

CARIDDI, L. N., M. L. GONZÁLEZ-PEREYRA, M. L. GAMBERO, M. S. DEMO, M. C. ISOLA, L. FRANZONI, L. I. SABINI \& A. M. MALDONADO. (2006). Minthostachys verticillata (Griseb.) Epling: A South American plant with anti-inflammatory effect in vitro. Allergy and Clinical Immunology International 18. https://doi.org./10.1027/0838-1925.18.5

CECHINEL FILHO, V. (2018). Marrubium vulgare L. En ALBUQUERQUE, U. P. \& U. PATIL \& A. MÁTHÉ (eds.), Medicinal and Aromatic Plants of South America, pp. 317-321. Springer, Dordrecht, Netherlands.

CERINI TREVISAN, S. C., A. PEREIRA PAES MENEZES, S. M. BARBALHO \& É. LANDGRAF GUIGUER. (2017). Properties of Mentha piperita. A brief review. World Journal of Pharmaceutical and Medical Research 3: 309-313.

CHISHTI, S., Z. A. KALOO \& P. SULTAN. (2013). Medicinal importance of genus Origanum: A review. Journal of Pharmacognosy and Phytotherapy 5: 170177. https://doi.org./10.5897/JPP2013.0285

DADÉ, M. M., D. E. FIORAVANTI, G. R. SCHINELLA \& H. A. TOURNIER. (2009). Total antioxidant capacity and polyphenol content of 21 aqueous extracts obtained from native plants of Traslasierra valley
(Argentina). Boletín Latinoamericano y del Caribe de Plantas Medicinales y Aromáticas 8: 529-539.

DAUQAN, E. M. A. \& A. ABDULLAH. (2017). Medicinal and functional values of Thyme (Thymus vulgaris L.) Herb. Journal of Applied Biology \& Biotechnology 5: 17-22. https://doi.org./10.7324/ JABB.2017.50203

DENNER, S. (2008). Lavandula angustifolia Mill. Holistic Nursing Practice 23: 57-64. https://doi.org./10.1097/01.HNP.0000343210.56710.fc

ELBAHNASAWY, A. S., E. R. VALEEVA, E. M. ELSAYED \& I. I. RAKHIMOV. (2019). The impact of thyme and rosemary on prevention of osteoporosis in rats. Journal of Nutrition and Metabolism 2019: 1431384. https://doi.org./10.1155/2019/1431384

ELHAJILI, M., K. BADDOURI K., S. ELKABBAJ, F. MEIOUAT \& A. SETTAF. (2001). Diuretic activity of the infusion of flowers from Lavandula officinalis. Reproduction Nutrition Development 41: 393-399.

ERDOGAN ORHAN, I., B. ÖZÇELIK, M. KARTAL \& Y. KAN. (2012). Antimicrobial and antiviral effects of essential oils from selected Umbelliferae and Labiatae plants and individual essential oil components. Turkish Journal of Biology 36: 239246. https://doi.org./10.3906/biy-0912-30

ESCOBAR, F. M., L. N. CARIDDI, M. C. SABINI, E. REINOSO, S. B. SUTIL, C. V. TORRES, S. M. ZANON \& L. I. SABINI. (2012). Lack of cytotoxic and genotoxic effects of Minthostachys verticillata essential oil: studies in vitro and in vivo. Food and Chemical Toxicology 50: 3062-3067. https://doi.org./10.1016/j.fct.2012.06.018

FATHIAZAD, F. \& S. HAMEDEYAZDAN. (2011). A review on Hyssopus officinalis L.: Composition and biological activities. African Journal of Pharmacy and Pharmacology 5: 1959-1966.

https://doi.org./10.5897/AJPP11.527

FIERASCU, I., C. E. DINU-PIRVU, R. C. FIERASCU, B. S. VELESCU, V. ANUTA, A. ORTAN \& V. JINGA. (2018). Phytochemical profile and biological activities of Satureja hortensis L.: A review of the last decade. Molecules 23: 2458. https://doi.org/10.3390/molecules 23102458

GARCÍA-BELTRÁN, J. M. \& M. A. ESTEBAN. (2016). Properties and applications of plants of Origanum sp. genus. SM Journal of Biology 2: 1006.

GEZICI, S. (2018). Promising anticancer activity of lavender (Lavandula angustifolia Mill.) essential oil through induction of both apoptosis and necrosis. Annals of Phytomedicine 7: 38-45. https://doi.org./10.21276/ap.2018.7.2.5 
GEORGIEV, Y. N., B. S. PAULSEN, H. KIYOHARA, M. CIZ, M. H. OGNYANOV, O. VASICEK, F. RISE, P. N. DENEV, H. YAMADA, A. LOJEK, V. KUSSOVSKI, H. BARSETT, A. KRASTANOV, I. Z. YANAKIEVA \& M. G. KRATCHANOVA. (2017). The common lavender (Lavandula angustifolia Mill.) pectic polysaccharides modulate phagocytic leukocytes and intestinal Peyer's patch cells. Carbohydrate Polymers 174: 948-959. https://doi.org./10.1016/j.carbpol.2017.07.011

GHORBANI, A. \& M. ESMAEILIZADEH. (2017). Pharmacological properties of Salvia officinalis and its components. Journal of Traditional and Complementary Medicine 7: 433-440. https://doi.org./10.1016/j.jtcme.2016.12.014

GUARDO, N. I., P. SAINZ, A. GONZÁLEZ-COLOMA, J. BURILLO \& R. A. MARTINEZ-DÍAZ. (2017). Trypanocidal effects of essential oils from selected medicinal plants. Synergy among the main components. Natural Product Communications 12: 709-712. https://doi.org/10.1177/1934578X1701200516

HAJIGHASEMI, F., V. HASHEMI \& F. KHOSHZABAN. (2011). Cytotoxic effect of Mentha spicata aqueous extract on cancerous cell lines in vitro. Journal of Medicinal Plant Research 5: 5142-5147.

HAMED, H., S. BOULILA, F. GHRAB, R. KALLEL, T. BOUDAWARA \& A. EL FEKI. (2018). The preventive effect of aqueous extract of Rosemary (Rosmarinus officinalis) leaves against the nephrotoxicity of carbon tetrachloride in mice. Archives of Physiology and Biochemistry 30: 1-8. https://doi.org./10.1080/13813455.2018.1508236

HASSANI, F. V., K. SHIRANI \& H. HOSSEINZADEH. (2016). Rosemary (Rosmarinus officinalis) as a potential therapeutic plant in metabolic syndrome: a review. Archiv für Experimentelle Pathologie und Pharmakologie 389: 931-949. https://doi.org./10.1007/s00210-016-1256-0

HEIDARI, Z., A. SALEHZADEH, S. A. SADAT SHANDIZ \& S. TAJDOOST. (2018). Anticancer and antioxidant properties of ethanolic leaf extract of Thymus vulgaris and its bio-functionalized silver nanoparticles. 3 Biotech 8: 177. https://doi.org./10.1007/s13205-018-1199-x

HOSSEINZADEH, S., A. JAFARI KUKHDAN, A. HOSSEINI \& R. ARMAND. (2015). The application of Thymus vulgaris in traditional and modern medicine: A review. Global Journal of Pharmacology 9: 260-266.

https://doi.org./10.5829/idosi.gjp.2015.9.3.94246
HURRELL, J. A. (2014). Urban Ethnobotany in Argentina: Theoretical advances and methodological strategies. Ethnobiology and Conservation 3: 2. https://doi.org./10.15451/ec2014-6-3.3-1-11

HURRELL, J. A. (2018). Clinopodium gilliesii (Benth.) Kuntze. En ALBUQUERQUE, U.P., U. PATIL \& A. MÁTHÉ (eds.), Medicinal and Aromatic Plants of South America, pp. 163-172. Springer, Dordrecht, Netherlands.

HURRELL, J.A. \& M. L. POCHETTINO. (2014). Urban Ethnobotany: theoretical and methodological contributions. En ALBUQUERQUE, U. P., L. V. F. CRUZ DA CUNHA, R. F. P. LUCENA \& R. R. N. ALVES (eds.), Methods and techniques in Ethnobiology and Ethnoecology, pp. 293-309. Springer, Berlin, Germany.

HURRELL, J. A. \& J. P. PUENTES. (2013). Medicinal and aromatic species of Asteraceae commercialized in the conurbation Buenos Aires-La Plata (Argentina). Ethnobiology and Conservation 2: 7. https://doi.org./10.15451/ec2013-8-2.7-1-40

HURRELL, J. A. \& J. P. PUENTES. (2017). Plant species and products of the Traditional Chinese Phytotherapy in the Ciudad Autónoma de Buenos Aires, Argentina. Ethnobiology and Conservation 6: 1. https://doi.org./10.15451/ec2017026.1143

HURRELL, J. A., E. A. ULIBARRI, P. M. ARENAS \& M. L. POChETTINO. (2011). Plantas de herboristería. Editorial Lola, Buenos Aires.

HURRELL, J. A., P. M. ARENAS \& M. L. POCHETTINO. (2013). Plantas de dietéticas. Editorial Lola, Buenos Aires.

HURRELL, J. A., P. M. ARENAS \& I. CRISTINA. (2015a). El conocimiento botánico en zonas urbanas: Potenciadores cognitivos comercializados en el Área Metropolitana de Buenos Aires, Argentina. Gaia Scientia 9: 17-31.

HURRELL, J. A., J. P. PUENTES \& P. M. ARENAS. (2015b). Medicinal plants with cholesterol-lowering effect marketed in the Buenos Aires-La Plata conurbation, Argentina: An Urban Ethnobotany study. Ethnobiology and Conservation 4: 6 .

https://doi.org./10.15451/ec2015-7-4.6-1-12

JAFARI, F., F. GHAVIDEL \& M. M. ZARSHENAS. (2016). A critical overview on the pharmacological and clinical aspects of popular Satureja species. Journal of Acupuncture and Meridian Studies 9: 118-127.

https://doi.org./10.1016/j.jams.2016.04.003

KANGWAN, N., K. PINTHA, S. LEKAWANVIJIT \& M. SUTTAJIT. (2019). Rosmarinic acid enriched 
fraction from Perilla frutescens leaves strongly protects indomethacin-induced gastric ulcer in rats. BioMed Research International 2019: 9514703. https://doi.org./10.1155/2019/9514703

KARGOZAR, R., H. AZIZI \& R. SALARI. (2017). A review of effective herbal medicines in controlling menopausal symptoms. Electronic Physician 9: 5826-5833. https://doi.org./10.19082/5826

KHAFAJI, S. S. O. (2018). Subject review: pharmacological application of thyme. Advances in Animal and Veterinary Sciences 6: 366-371. https://doi.org./10.17582/journal.aavs/2018/6.8

KHAIR-UL-BARIYAH, S., D. AHMED \& M. IKRAM. (2012). Ocimum basilicum: A review on phytochemical and pharmacological studies. Pakistan Journal of Chemistry 2: 78-85. https://doi: 10.15228/2012.v02.i02.p05.

KEDIA, A., B. PRAKASH, P. KUMAR MISHRA, C. S. CHANOTIYA \& N. K. DUBEY. (2014). Antifungal, antiaflatoxigenic, and insecticidal efficacy of spearmint (Mentha spicata L.) essential oil. International Biodeterioration and Biodegradation 89: 29-36. https://doi.org./10.1016/j.ibiod.2013.10.027

KOULIVAND, P. H., M. K. GHADIRI \& A. GORJI. (2013). Lavander and the Nervous System. EvidenceBased Complementary and Alternative Medicine 2013: 681304. https://doi.org./10.1155/2013/681304

LIMONCU, M. E., C. BALCIOĞLU, T. OYUR, G., ZEYBEK \& U. ZEYBEK. (2017). In vitro investigation of the pediculicidal activities of the volatile oil components of some medical plants raised in Turkey. Turkiye Parazitolojii Dergisi 41: 208-213. https://doi.org./10.5152/tpd.2017.5201

LOPRESTI, A. L. (2017). Salvia (Sage): A review of its potential cognitive-enhancing and protective effects. Drugs in R\&D 17: 53-64.

https://doi.org./10.1007/s40268-016-0157-5

LOOLAIE, M., N. MOASEFI, H. RASOULI \& H. ADIBI. (2017). Peppermint and its functionality: A review. Archives of Clinical Microbiology 8: 54. https://doi.org./10.4172/1989-8436.100053

MOUSSOUNI, L., M. BENHANIFIA \& A. AYAD. (2018). In-vitro anthelmintic effects of aqueous and ethanolic extracts of Marrubium vulgare leaves against bovine digestive strongyles. Turkiye Parazitolojii Dergisi 42: 262-267. https://doi.org./10.5152/tpd.2018.5972

ONIGA, I. C. PUȘCAȘ, R. SILAGHI-DUMITRESCU, N. K. OLAH, B. SEVASTRE, R. MARICA, I. MARCUS, A. C. SEVASTRE-BERGHIAN, D. BENEDEC, C. E. POP \& D. HANGANU.
(2018). Origanum vulgare ssp. vulgare: chemical composition and biological studies. Molecules 23, pii: E2077. https://doi.org./10.3390/molecules23082077

PAUNOVIC, V., M. KOSIC, S. DJORDJEVIC, A. ZUGIC, N. DJALINAC, U. GASIC, V. TRAJKOVIC \& J. HARHAJI-TRAJKOVIC. (2016). Marrubium vulgare ethanolic extract induces proliferation block, apoptosis, and cytoprotective autophagy in cancer cells in vitro. Cellular and Molecular Biology (Noisy-le-Grand, France) 62: 108-114. https://doi.org./10.14715/cmb/2016.62.11.18

PRASANTH REDDY, V., K. RAVI VITAL, P. V. VARSHA\& S. SATYAM. (2014). Review on Thymus vulgaris. Traditional uses and pharmacological properties. Medicinal and Aromatic Plants 3: 164. https://doi.org./10.4172/2167-0412.1000164

PRERNA, S. \& N. VASUDEVA. (2015). Origanum majorana L. Phyto-pharmacological review. Indian Journal of Natural Products and Resources 6: 261-267.

PUBMED. (2019). NCBI, US National Library of Medicine. National Institute of Health. Disponible: https://www.ncbi.nlm.nih.gov/pubmed/ (Consulta: III-2019).

PUENTES, J. P. (2016). Plantas medicinales y productos derivados comercializados como antidiabéticos en la conurbación Buenos Aires-La Plata, Argentina. Boletín Latinoamericano y del Caribe de Plantas Medicinales y Aromáticas 15: 373-397.

PUENTES, J. P. (2017). Etnobotánica urbana: el conocimiento botánico local sobre las plantas alimenticias y medicinales, y sus usos, en la conurbación Buenos Aires-La Plata. Tesis Doctoral. Facultad de Ciencias Naturales y Museo, Universidad Nacional de La Plata, Argentina.

PUENTES, J. P., P. M. ARENAS \& J. A. HURRELL. (2018). Productos medicinales y condimenticios de Lamiaceae y Verbenaceae comercializados en el Área Metropolitana Buenos Aires-La Plata, Argentina. Dominguezia 34 (Supl.): 32-33.

RASOOL, R. \& B. A. GANAI. (2013). Prunella vulgaris L.: A literature review on its therapeutic potentials. Pharmacologia 4: 441-448. https://doi.org./10.5567/pharmacologia.2013.441.448 RITA, P. \& D. K. ANIMESH. (2011). An updated overview on peppermint (Mentha piperita). International Research Journal of Pharmacy 2: 1-10. RODRÍGUEZ VILLANUEVA, J. \& J. MARTÍN ESTEBAN. (2016). An Insight into a Blockbuster Phytomedicine; Marrubium vulgare L. Herb. More of a Myth than a Reality? Phytotherapy Research 30: 1551-1558. https://doi.org./10.1002/ptr.5661 
ROSSI, Y. E., L. CANAVOSO \& S. M. PALACIOS. (2012). Molecular response of Musca domestica L. to Mintostachys verticillata essential oil, (4R) (+)-pulegone and menthone. Fitoterapia 83: 336342. https://doi.org./10.1016/j.fitote.2011.11.019

SALEHI, A. \& M. SETORKI. (2017). Effect of Hyssopus officinalis essential oil on chronic stress induced memory and learning impairment in male mice. Bangladesh Journal of Pharmacology 12: 448-454. https://doi.org/10.3329/bjp.v12i4.33585

SHANG, X., P. HU, X. WANG, H. HE \& L. MAOXING. (2014). Leonurus japonicus Houtt.: Ethnopharmacology, phytochemistry and pharmacology of an important traditional Chinese medicine. Journal of Ethnopharmacology 152: 1432. https://doi.org./10.1016/j.jep.2013.12.052

SESTILI, P., T. ISMAIL, C. CALCABRINI, M. GUESCINI, E. CATANZARO, E. TURRINI, A. LAYLA, S. AKHTAR \& C. FIMOGNARI. (2018). The potential effects of Ocimum basilicum on health: a review of pharmacological and toxicological studies. Expert Opinion on Drug Metabolism and Toxicology 2018. https://doi.org./10.1080/17425255.2018.1484450

SHAKERI, A., A. SAHEBKAR \& B. JAVADI. (2016). Melissa officinalis L. A review of its traditional uses, phytochemistry and pharmacology. Journal of Ethnopharmacology 188: 204-228. https://doi.org./10.1016/j.jep.2016.05.010

SHARMA, A., G. A. NAYIK \& D. S. CANNOO. (2019). Pharmacology and toxicology of Nepeta cataria (Catmint) species of genus Nepeta: A review. In OZTURK, M. \& K. R. HAKEEM (eds.), Plant and Human Health, Vol. 3, pp. 285-299. Springer Nature, Cham, Switzerland.

SINGH, P., P. KOTHIYAL \& P. RATAN. (2018). Pharmacological and phytochemical studies of Origanum vulgare: A review. International Research Journal of Pharmacy 9. https://doi.org./10.7897/2230-8407.09685

SINGLETARY, K. W. (2010). Oregano: Overview of the literature on health benefits. Nutrition Today 45: 129-138.

https://doi.org./10.1097/NT.0b013e3181dec789

SOURI, F., K. RAKHSHAN, S. ERFANI, Y. AZIZI, S. NASSERI MALEKI \& N. ABOUTALEB. (2018). Natural lavender oil (Lavandula angustifolia) exerts cardioprotective effects against myocardial infarction by targeting inflammation and oxidative stress. Inflammopharmacology 2018. https://doi.org./10.1007/s10787-018-0520-y
STAPPEN, I., J. WANNER, N. TABANCA, D. E. WEDGE, A. ALI, V. K. KAUL, B. LAL, V. JAITAK, V. K. GOCHEV, E. SCHMIDT \& L. JIROVETZ. (2015). Chemical composition and biological activity of essential oils of Dracocephalum heterophyllum and Hyssopus officinalis from Western Himalaya. Natural Product Communications 10: 133-138. https://doi.org/10.1177/1934578X1501000131

ŠTEFANIDESOVÁ, K., L. ŠKULTÉTY, O. A. E. SPARAGANO \& E. ŠPITALSKÁ. (2017). The repellent efficacy of eleven essential oils against adult Dermacentor reticulatus ticks. Ticks and Tickborne Diseases 8: 780-786. https://doi.org./10.1016/j.ttbdis.2017.06.003

TAHIR, M., M. KHUSHTAR, M. FAHAD \& M. D. AZIZUR RAHMAN. (2018). Phytochemistry and pharmacological profile of traditionally used medicinal plant Hyssop (Hyssopus officinalis L.). Journal of Applied Pharmaceutical Science 8: 132140. https://doi.org./10.7324/JAPS.2018.8721

TEPE, B. \& M. CILKIZ. (2015). A pharmacological and phytochemical overview on Satureja. Pharmaceutical Biology 54: 375-412. https://doi.org./10.3109/13880209.2015.1043560

THE PLANT LIST. (2013). The Plant List. Version 1.1. Disponible: http://www.theplantlist.org/ (Consulta: IV-2019).

TRIPATHY, B., S. SATYANARAYANA, K. ABEDULLA KHAN \& K. RAJA. (2017). An updated review on traditional uses, taxonomy, phytochemistry, pharmacology and toxicology of Origanum majorana. International Journal of Pharma Research and Health Sciences 5: 1717-1723. https://doi.org./10.21276/ijprhs.2017.04.01

ULBRICHT, C., W. CHAO, K. NUMMY, E. RUSIE, S. TANGUAY-COLUCCI, C. M. IANNUZZI, J. B. PLAMMOOTTIL, M. VARGHESE \& W. WEISSNER. (2009). Chia (Salvia hispanica): a systematic review by the natural standard research collaboration. Reviews on Recent Clinical Trials 4: 168-174.

https://doi.org./10.2174/157488709789957709

ULBRICHT, C., D. COSTA, J. M. GRIMES SERRANO, J. GUILFORD, R. ISAAC, E. SEAMON \& M. VARGHESE. (2010). An evidence-based systematic review of spearmint by the natural standard research collaboration. Journal of Dietary Supplements 7: 179-215.

https://doi.org./10.3109/19390211.2010.486702

Ullah, R., M. NADEEM, A. KHALIQUE, M. IMRAN, S. MEHMOOD, A. JAVID \& J. 
HUSSAIN. (2016). Nutritional and therapeutic perspectives of Chia (Salvia hispanica L.): a review. Journal of Food Science and Technology 53: $1750-1758$.

https://doi.org./10.1007/s13197-015-1967-0

VALDIVIA-LÓPEZ, M. Á. \& A. TECANTE. (2015).

Chia (Salvia hispanica): A review of native Mexican seed and its nutritional and functional properties. Advances in Food and Nutrition Research 75: 53-75.

https://doi.org./10.1016/bs.afnr.2015.06.002
YU, H., J. F. QIU, L. J. MA, Y. J. HU, P. LI \& J. B. WAN. (2017). Phytochemical and phytopharmacological review of Perilla frutescens L. (Labiatae), a traditional edible-medicinal herb in China. Food and Chemical Toxicology 108 (Pt B): 375-391. https://doi.org./10.1016/j.fct.2016.11.023

ZOLFAGHARIAN, F., B. M. RAZAVI \& H. HOSSEINZADEH. (2016). Anticonvulsant effect of Satureja hortensis aerial parts extracts in mice. Avicenna Journal of Phytomedicine 6: 305-312. https://doi.org./10.22038/ajp.2016.5273 